\title{
Metales pesados en sedimentos de la Laguna de Bustillos, Chihuahua, México y comparación de agua regia y peróxido de hidrógeno como métodos de digestión
}

\author{
Heavy metals in sediments of the Bustillos Lagoon, Chihuahua, Mexico \\ and comparison of agua regia and hydrogen peroxide as digestion methods

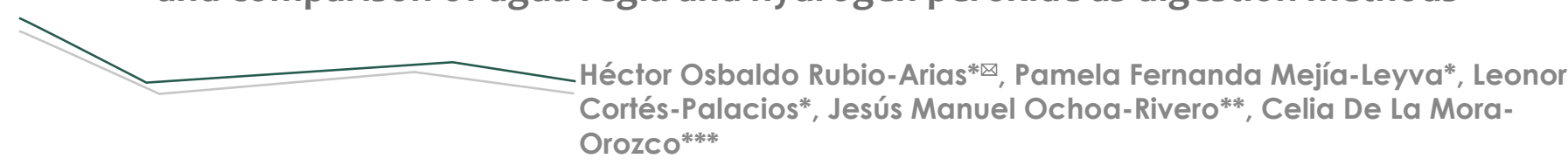 \\ Rubio-Arias, H. O., Mejía-Leyva, P. F., Cortés-Palacios, L., Ochoa-Rivero, J. M., \& De La \\ Mora-Orozco, C. Metales pesados en sedimentos de la Laguna de Bustillos, Chihuahua, \\ México y comparación de agua regia y peróxido de hidrógeno como métodos de \\ digestión. Investigación y Ciencia de la Universidad Autónoma de Aguascalientes, \\ 26(74), 39-47.
}

\section{RESUMEN}

El objetivo fue conocer la contaminación por metales en sedimentos de la Laguna de Bustillos y comparar dos métodos de digestión (agua regia y peróxido). Se obtuvieron muestras en 15 puntos aleatorios de la superficie $(0-10 \mathrm{~cm})$ y de la parte inferior (10-20 $\mathrm{cm})$. El análisis se realizó en un ICP-OES cuantificando cromo ( $\mathrm{Cr})$, fierro (Fe), litio (Li), magnesio (Mg), manganeso (Mn), níquel (Ni), zinc ( $\mathrm{Zn})$, cobalto (Co), cobre (Cu), plomo (Pb) y estroncio (Sr). Se efectuó un ANOVA bajo un arreglo factorial $2 \times 2$. Los resultados mostraron diferencias por el método de digestión $(P<0.05)$, pero no por profundidad $(P>0.05)$ o la interacción $(P>0.05)$. La concentración de $\mathrm{Cr}$, Fe, $\mathrm{Mn}, \mathrm{Ni}, \mathrm{Zn}, \mathrm{Cu}$ y Pb no superó las normas nacionales o internacionales y para Li, Mg, Co y Sr no existen estándares. Se concluye que el sedimento se encuen-

Palabras clave: sedimentación; polución; elementos pesados; ecosistema acuático.

Keywords: sedimentation; pollution; heavy metals; aquatic ecosystem.

Recibido: 10 de junio de 2017, aceptado: 12 de enero de 2018

* Facultad de Zootecnia y Ecología, Universidad Autónoma de Chihuahua. Periférico Francisco R. Almada km 1 carretera Chihuahua-Cuauhtémoc, Colonia Zootecnia, C. P. 31031, Chihuahua, México. Correo electrónico: rubioa1105@hotmail.com; pfernanda.mejia@gmail.com; Icortes@uach.mx

** Instituto Nacional de Investigaciones Forestales, Agrícolas y Pecuarias campo experimental La Campana. Km 33.3 carretera Chihuahua-Ojinaga, Aldama, C. P. 32910, Chihuahua, México. Correo electrónico: chuy-8-a@hotmail.com

*** Instituto Nacional de Investigaciones Forestales, Agrícolas y Pecuarias campo experimental Centro Altos de Jalisco. Km. 8 carretera libre Tepatitlán-Lagos de Moreno, Tepatitlán de Morelos, C. P. 47600, Jalisco, México. Correo electrónico: delamora.celia@inifap.gob.mx

$凶$ Autor para correspondencia tra con niveles de metales que no representan un peligro para el ecosistema.

ABSTRACT

The aim was to quantify the level of pollution with metals in sediments of the Bustillos Lagoon and to compare two methods of digestion (agua regia and peroxide). Fifteen random points were selected, and samples were collected at the top $(0-10 \mathrm{~cm})$ and below $(10-20 \mathrm{~cm})$. The analysis was performed in a ICPOES quantifying chromium ( $\mathrm{Cr}$ ), iron (Fe), lithium (Li), magnesium (Mg), manganese ( $\mathrm{Mn})$, nickel (Ni), zinc $(\mathrm{Zn})$, cobalt (Co), cooper (Cu), lead (Pb) and strontium (Sr). An ANOVA was carried out considering a $2 \times 2$ factorial arrangement. The results showed statistical differences for the digestion method $(P<0.05)$, but no differences was noted for depth $(P>0.05)$ neither for the interaction $(P>0.05)$. It is concluded that the sediments of the Bustillos ecosystem do not represent a hazard because the metals $\mathrm{Cr}, \mathrm{Fe}, \mathrm{Mn}$, $\mathrm{Ni}, \mathrm{Zn}, \mathrm{Cu}$ and $\mathrm{Pb}$ were not above the international and national standards and the metals $\mathrm{Li}, \mathrm{Mg}$, Co and $\mathrm{Sr}$ do not have standards.

\section{INTRODUCCIÓN}

El nombre genérico de sedimentos se utiliza con respecto a partículas de rocas o suelos que son transportadas por diversos mecanismos y luego sedimentan. Estos materiales son depositados en lagos, ríos, presas, así como en planicies, e incluso, en el mar (White et al., 2007). Los sedimentos pueden provenir de una fuente natural, o bien, de un efecto antropogénico. Por ejemplo, la erosión es considera- 
IIVESTIGACIÓn Y CIERCIA DE LA UNIVERSIDAD AUTÓNOMA

40 DE RGUASCALIERTES da como una fuente natural debido al proceso de deslizamiento de materiales, mientras que un efecto antropogénico lo representa un cambio de uso del suelo, sitios de construcción, así como desechos mineros, urbanos e industriales realizados por el hombre (García \& Maza, 1998; Lin, Liu, Zhang, Li, \& Shen, 2016; Zhang, Wang, Ali, \& DeLaune, 2016), lo que en consecuencia, incrementa el nivel de sedimentos y ocasiona una amenaza para los ecosistemas y la humanidad (Alemayehu, 2006; CCME, 2002).

Los metales presentan una alta densidad y suelen ser tóxicos o venenosos, incluso en concentraciones bajas. Esta peligrosidad se explica por el hecho de no ser degradables, ni química, ni biológicamente. Una vez en el ambiente pueden permanecer largos periodos de tiempo, representar un gran peligro y alto riesgo, y causar la destrucción de fauna y flora.

Entre los metales considerados más riesgosos, desde un punto de vista ambiental, se encuentran el mercurio (Hg), cadmio (Cd), zinc ( $\mathrm{Zn})$, cobre (Cu) y plomo (Pb) (Páez-Osuna, 1996). Otros, como el fierro (Fe) y el manganeso ( $\mathrm{Mn}$ ) son elementos considerados como esenciales; no obstante, cuando se tiene una alta concentración, los organismos experimentan algún grado de estrés y disminuyen sus posibilidades de sobrevivir (Chen et al., 2017). En general, se ha demostrado que tanto los metales como algunos compuestos (p. e. orgánicos) pueden ser removilizados por un efecto de bioperturbación y/o resuspensión (Sprovieri et al., 2007). Zhang et al. (2016) mencionaron que los sedimentos tienen la particularidad de reunir metales y representan un indicador del nivel de contaminación de un ecosistema acuático.

La Laguna de Bustillos, en el norte de México, ha sido considerada como un ícono ecológico que presenta una problemática de modificación por efectos de la deforestación de su cuenca, la desecación por una sobreexplotación de los mantos freáticos y contaminación por acarreo de agroquímicos, desechos sólidos y aguas residuales urbanas (Ochoa-Rivero et al., 2017; Rubio Arias et al., 2006). Estudios previos han demostrado que el agua está contaminada (Rubio Arias, Saucedo, Lara, Wood, \& Jiménez, 2005a) y sus peces presentan altos niveles de $\mathrm{Zn}$ y Sr en agallas y músculo (Rodríguez, 2013). En otro estudio, Amado, Ortiz, Solano, Orozco y Ramírez (1991) clasificaron el agua como C3S3, la cual no es recomendable para cultivos agrícolas, debido al alto contenido de sales, de carbonatos de sodio y de elementos tóxicos. Un agua tipo C3S3 contiene un nivel de salinidad entre 750 y $2,200 \mu S \mathrm{~cm}^{-1} \mathrm{y}$, en consecuencia, es peligrosa.

El objetivo fue cuantificar el nivel de contaminación por metales en sedimentos de la Laguna de Bustillos en Chihuahua, México, y comparar el método de agua regia y el peróxido de hidrógeno como técnicas de digestión. La hipótesis fue que, debido a que el agua se encuentra contaminada, los sedimentos también contienen niveles que pueden representar un problema para el ecosistema. Una segunda hipótesis fue que existen diferencias entre métodos de digestión, por lo que es necesario cuantificar esas diferencias. El proceso de digestión permite transformar los metales a una forma líquida para su posterior cuantificación. Estos resultados aportarán elementos para la predicción o corrección de este ecosistema acuático, y sugerirán futuras investigaciones para la utilización del mejor método de digestión.

MATERIALES Y MÉTODOS

La presente investigación se condujo en la Laguna de Bustillos, que se localiza en el municipio de Cuauhtémoc, Chihuahua, México (figura 1). Este cuerpo de agua natural cuenta con una longitud de 25 a 30 $\mathrm{km}$ y un ancho de $4 \mathrm{~km}$ y se encuentra en las coordenadas de latitud $28^{\circ} 28^{\prime} 48^{\prime \prime} \mathrm{N}$, longitud $106^{\circ} 44^{\prime} 39^{\prime \prime}$ O y una altitud de 1,992 m.s.n.m. Es un ecosistema endorreico localizado en la región hidrográfica de Cuencas Cerradas del Norte y donde la subcuenca presenta flujos virtuales, no perennes de agua. Los usos de suelo que se pueden encontrar en sus alrededores son bosques de encino y pino, pastizales inducidos y natural halófitos y tierras destinadas a la agricultura de riego y temporal, a las cuales se les puede atribuir parte de la contaminación que se presenta en la laguna (CONAGUA, s. f.).

Las muestras de sedimentos se obtuvieron los días 21 y 22 de mayo de 2013. De modo previo a la toma de muestras, en gabinete se seleccionaron 15 puntos aleatorios, obtenidos al cuadricular el área total utilizando imágenes satelitales en el programa Google ${ }^{T M}$ Earth y ArcGIS ${ }^{\circledR}$. En cada sitio seleccionado se tomaron dos muestras de sedimento $(2 \mathrm{~kg})$; en la parte superficial $(0-10 \mathrm{~cm})$ y en la parte inferior (10-20 cm). Las muestras se depositaron en plastibolsas, se identificaron y fueron trasladadas al laboratorio de parámetros ambientales de la Facultad de 


\section{IIVESTIGAGIÓn Y CUERCIA DE LA UกIVERSIDAD AUTÓOOOMA DE RGUASCALIERTES}

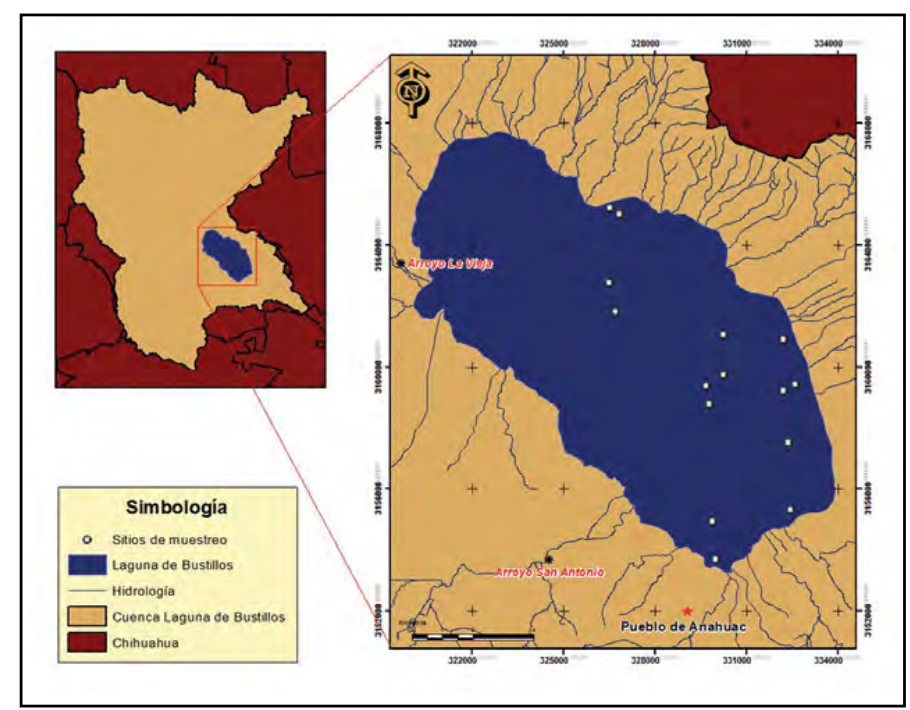

Figura 1. Localización del sistema acuático Laguna de Bustillos en el estado de Chihuahua, México.

Elaboración propia.

Zootecnia y Ecología de la Universidad Autónoma de Chihuahua. Las muestras se secaron a temperatura ambiente, asegurándose que la temperatura no excediera los $35^{\circ} \mathrm{C}$ y una humedad relativa de 30 y $70 \%$.

\section{Análisis de metales pesados}

Para el análisis de las muestras se realizó un tamizado utilizando una criba de $2 \mathrm{~mm}$ y se pesó $1 \mathrm{~g}$ de la muestra. Se emplearon dos métodos para la digestión; en el primero se utilizó agua regia, donde a la muestra se le agregaron $7.5 \mathrm{ml}$ de ácido clorhídrico concentrado ( $\mathrm{HCl}-\mathrm{C})$ y $2.5 \mathrm{ml}$ de ácido nítrico concentrado $\left(\mathrm{HNO}_{3}-\mathrm{C}\right)$, y se dejó reposar por $24 \mathrm{~h}$. Se llevaron a reflujo hasta un volumen de $5 \mathrm{ml}$, y se le agregaron nuevamente $7.5 \mathrm{ml}$ de HCL-C y $2.5 \mathrm{ml}$ de $\mathrm{HNO}_{3}-\mathrm{C}$, continuando el reflujo hasta un volumen de $5 \mathrm{ml}$.

El segundo método fue con peróxido de hidrógeno $\left(\mathrm{H}_{2} \mathrm{O}_{2}\right)$, donde se agregaron $10 \mathrm{ml}$ de $\mathrm{HNO}_{3}-\mathrm{C}$. Los matraces se taparon con un vidrio de reloj y se calentaron durante 15 min hasta hervor, se dejaron reposar hasta alcanzar la temperatura ambiente. Se procedió a agregarles $5 \mathrm{ml}$ de $\mathrm{HNO}_{3}-\mathrm{C}$ hasta que dejaron de reaccionar. Se adicionaron $2 \mathrm{ml}$ de agua tridestilada y $3 \mathrm{ml}$ de $\mathrm{H}_{2} \mathrm{O}_{2}$ para que reaccionaran, se agregaron $2 \mathrm{ml}$ de $\mathrm{H}_{2} \mathrm{O}_{2}$ y se procedió al filtrado, para lo cual se colocó papel filtro sobre embudos y sobre el papel se agregó agua tridestilada. Una vez realizadas las digestiones, las muestras se analizaron en un espectrofotómetro de emisión óptica por plasma acoplado inductivamente (ICP-OES), y se cuantificaron cromo (Cr), fierro (Fe), litio (Li), magnesio (Mg), manganeso (Mn), níquel (Ni), zinc (Zn), cobalto (Co), cobre (Cu), plomo (Pb) y estroncio (Sr).

\section{Análisis estadístico}

Se implementó un análisis de varianza (ANOVA) considerando un diseño de tratamientos factorial 2x2. El factor A fue la profundidad con dos niveles; superior $(0-10 \mathrm{~cm})$ e inferior $(10-20 \mathrm{~cm})$. El factor $B$ fue el método de digestión con dos niveles; agua regia y con peróxido. Se graficaron efectos mayores y de interacción para visualizar los datos obtenidos, como lo sugieren Rubio Arias y Jiménez (2012). El modelo estadístico utilizado fue:

$$
Y_{i j k}=\mu+\alpha i+\beta j+(\alpha \beta)_{i j}+\epsilon_{i j k^{\prime}} \text { con } i=1,2 ; j=1,2
$$

donde:

$Y_{i j k}=\quad$ Respuesta de la k-ésima repetición en el i-ésimo nivel del factor $A$ y j-ésimo nivel del factor B

$\mu=\quad$ Media General

$\alpha i=$ Efecto del i-ésimo nivel del factor $A$

$\beta \mathrm{j}=\quad$ Efecto del $\mathrm{j}$-ésimo nivel del factor $B$

$(\alpha \beta)_{i j}=$ Efecto de interacción entre factor A y factor B

$\epsilon_{i \mathrm{ik}}=\quad$ Error asociado a la observación ijk-ésima

\section{RESULTADOS}

El Cr fue estadísticamente diferente por el factor método $(P<0.05)$, pero no para la profundidad $(P>0.05)$ ni para la interacción $(P>0.05)$. La media con peróxido fue de $8.79 \mathrm{mg} \mathrm{kg}^{-1}$ (figura 2) y con agua regia fue de $1.198 \mathrm{mg} \mathrm{kg}^{-1}$ (figura 3), mientras que para la profundidad de $0.10 \mathrm{~m}$ fue de $4.98 \mathrm{mg} \mathrm{kg}-1$ y para la de $0.20 \mathrm{~m}_{\text {fue de }} 5.01 \mathrm{mg} \mathrm{kg}^{-1}$ (figura 4). El Fe fue estadísticamente diferente por el factor método $(P<0.05)$, pero no para la profundidad $(P>0.05) \mathrm{ni}$ para la interacción $(P>0.05)$. La media con peróxido fue de 4,408 $\mathrm{mg} \mathrm{kg}^{-1}$ y con agua regia de $2,082 \mathrm{mg}$ $\mathrm{kg}^{-1}$, mientras que para la profundidad de $0.10 \mathrm{~m}$ fue de $3,174 \mathrm{mg} \mathrm{kg}^{-1}$ y para la de $0.20 \mathrm{~m}$ fue de $3,315 \mathrm{mg}$ $\mathrm{kg}^{-1}$ (figura 4).

El Li fue estadísticamente diferente por el factor método $(P<0.05)$, pero no para la profundidad $(P>0.05)$ ni para la interacción $(P>0.05)$. La media con peróxido fue de $9.42 \mathrm{mg} \mathrm{kg}^{-1}$ y con agua regia fue de $3.96 \mathrm{mg} \mathrm{kg}^{-1}$, mientras que para la profundidad de $0.10 \mathrm{~m}$ fue de $7.21 \mathrm{mg} \mathrm{kg}^{-1}$ y para la de 0.20 $\mathrm{m}$ fue de $6.17 \mathrm{mg} \mathrm{kg}^{-1}$ (figura 4). El Mg fue estadísticamente diferente por el factor método $(P<0.05)$, 
IIVESTIGACIÓn Y CIERCIA DE LA UחIVERSIDAD AUTÓNOMH

42

\section{DE AGUASCALIERTES}

pero no para la profundidad $(P>0.05)$ ni para la interacción $(P>0.05)$. La media con peróxido fue de $2,092 \mathrm{mg} \mathrm{kg}^{-1}$ y con agua regia fue de $1,424 \mathrm{mg} \mathrm{kg}^{-1}$, mientras que para la profundidad de $0.10 \mathrm{~m}$ fue de $1,823 \mathrm{mg} \mathrm{kg}^{-1}$ y para la de $0.20 \mathrm{~m}$ fue de $1,692 \mathrm{mg}$ kg-1 (figura 4).

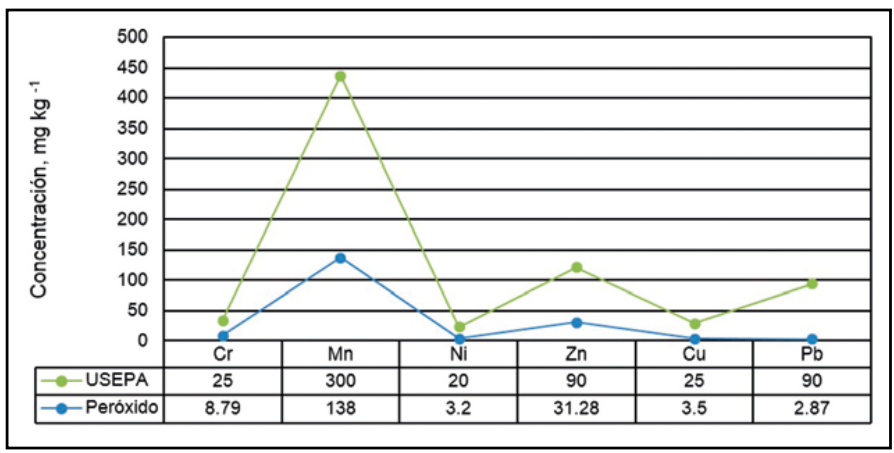

Figura 2. Medias de metales pesados cuantificados con el método de peróxido y los límites establecidos por la US EPA.

Elaboración propia.

El Mn fue estadísticamente diferente por el factor método $(P<0.05)$, pero no para la profundidad $(P>0.05)$ ni para la interacción $(P>0.05)$. La media con peróxido fue de $138 \mathrm{mg} \mathrm{kg}^{-1}$ (figura 2) y con agua regia fue de $87.85 \mathrm{mg} \mathrm{kg}^{-1}$ (figura 3), mientras que para la profundidad de $0.10 \mathrm{~m}$ fue de $109.2 \mathrm{mg}$ $\mathrm{kg}^{-1}$ y para la de $0.20 \mathrm{~m}$ fue de $116.7 \mathrm{mg} \mathrm{kg}^{-1}$ (figura 4). El Ni fue estadísticamente diferente por el factor método $(P<0.05)$, pero no para la profundidad $(P>0.05)$ ni para la interacción ( $P>0.05)$. La media con peróxido fue de $3.20 \mathrm{mg} \mathrm{kg}^{-1}$ (figura 2) y con agua regia fue de $0.499 \mathrm{mg} \mathrm{kg}^{-1}$ (figura 4), mientras que para la profundidad de $0.10 \mathrm{~m}$ fue de $1.777 \mathrm{mg} \mathrm{kg}^{-1}$ y para la de $0.20 \mathrm{~m}_{\text {fue de }} 1.923 \mathrm{mg} \mathrm{kg}^{-1}$ (figura 4).

El Zn fue estadísticamente diferente por el factor método $(P<0.05)$, pero no para la profundidad $(P>0.05)$ ni para la interacción $(P>0.05)$. La media con peróxido fue de $31.28 \mathrm{mg} \mathrm{kg}^{-1}$ (figura 2) y con agua regia de $6.77 \mathrm{mg} \mathrm{kg}^{-1}$ (figura 3), mientras que para la profundidad de $0.10 \mathrm{~m}$ fue de $21.92 \mathrm{mg} \mathrm{kg}^{-1}$ y para la de $0.20 \mathrm{~m}$ fue de $16.14 \mathrm{mg} \mathrm{kg}^{-1}$ (figura 4). El Co no fue estadísticamente diferente por el factor método $(P<0.05)$ ni para la profundidad $(P>0.05)$ ni la interacción $(P>0.05)$. La media con peróxido fue de $0.726 \mathrm{mg} \mathrm{kg}^{-1}$ y con agua regia fue de $0.2750 \mathrm{mg}$ $\mathrm{kg}-1$, mientras que para la profundidad de $0.10 \mathrm{~m}$ fue de $0.554 \mathrm{mg} \mathrm{kg}^{-1}$ y para la de $0.20 \mathrm{~m}$ fue de 0.477 $\mathrm{mg} \mathrm{kg}^{-1}$ (figura 4 ).

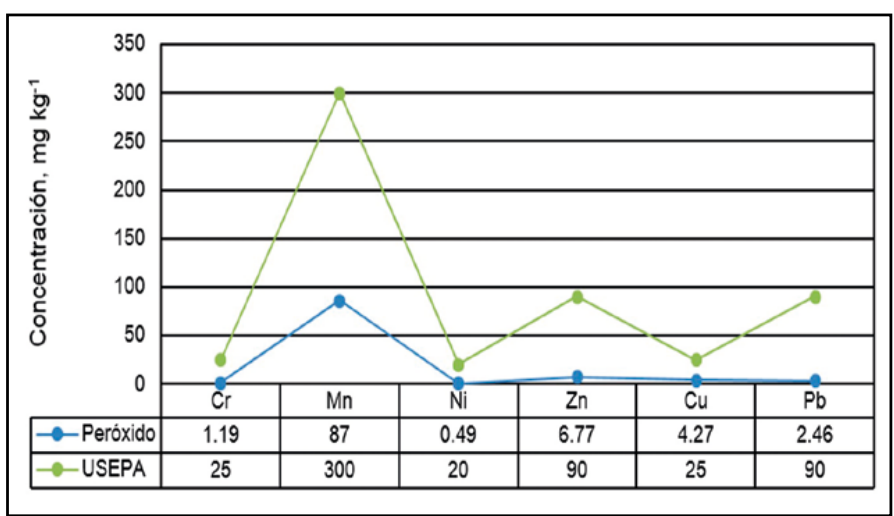

Figura 3. Medias de metales pesados cuantificadas por el método de agua regia y los límites establecidos por la US EPA. Elaboración propia.

El Cu no fue estadísticamente diferente por el factor método $(P>0.05)$ ni para la profundidad $(P>0.05)$ ni la interacción $(P>0.05)$. La media con peróxido fue de $3.504 \mathrm{mg} \mathrm{kg}^{-1}$ (figura 2) y con agua regia fue de $4.27 \mathrm{mg} \mathrm{kg}^{-1}$ (figura 3), mientras que para la profundidad de $0.10 \mathrm{~m}$ fue de $2.833 \mathrm{mg} \mathrm{kg}^{-1}$ y para la de $0.20 \mathrm{~m}$ fue de $4.94 \mathrm{mg} \mathrm{kg}^{-1}$ (figura 4). El Pb no fue diferente por el factor método $(P>0.05)$ ni para la profundidad $(P>0.05)$ ni la interacción $(P>0.05)$. La media con peróxido fue de $2.872 \mathrm{mg} \mathrm{kg}^{-1}$ (figura 2) y con agua regia fue de $2.466 \mathrm{mg} \mathrm{kg}^{-1}$ (figura 3), mientras que para la profundidad de $0.10 \mathrm{~m}$ fue de $2.441 \mathrm{mg} \mathrm{kg}^{-1}$ y para la de $0.20 \mathrm{~m}$ fue de $2.897 \mathrm{mg}$ $\mathrm{kg}^{-1}$ (figura 4). El Sr no fue diferente por el factor método $(P>0.05)$ ni para la profundidad $(P>0.05)$ ni la interacción $(P>0.05)$. La media con peróxido fue de $74.76 \mathrm{mg} \mathrm{kg}^{-1}$ y con agua regia fue de $56.24 \mathrm{mg} \mathrm{kg}^{-1}$, mientras que para la profundidad de $0.10 \mathrm{~m}$ fue de $68.31 \mathrm{mg} \mathrm{kg}^{-1}$ y para la de $0.20 \mathrm{~m}$ fue de $62.69 \mathrm{mg}$ $\mathrm{kg}^{-1}$ (figura 4).

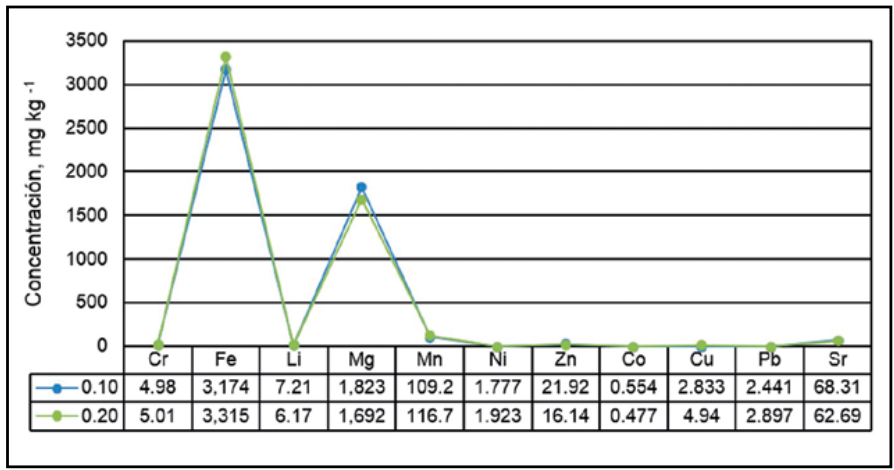

Figura 4. Medias de metales pesados en sedimentos cuantificados a dos profundidades.

Elaboración propia. 
DISCUSIÓN

Para el Cr en un estudio similar realizado en el Lago Pontchartrain en los Estados Unidos de Norteamérica (EE. UU.), los investigadores Zhang et al. (2016) reportaron valores en sedimentos en un rango de 5.32 a $13.14 \mathrm{mg} \mathrm{kg}^{-1}$ y utilizaron para el proceso de digestión de metales $\mathrm{HNO}_{3} \mathrm{HCl}$ y $\mathrm{H}_{2} \mathrm{O}_{2}$. En este estudio en particular las concentraciones no rebasaron los criterios establecidos por la US EPA (United States Environmental Protection Agency), ya que quedaron dentro del rango de "no contaminado": hasta $25 \mathrm{mg} \mathrm{kg}^{-1}$. Además, al considerar las concentraciones de referencia totales $\left(\mathrm{CR}_{\mathrm{T}}\right)$ para remediación de suelo para uso agrícola/residencial en la NOM147-SEMARNAT/SSA1-2004 (SEMARNAT, 2007) los niveles no sobrepasaron la referencia de $280 \mathrm{mg} \mathrm{kg}^{-1}$, por lo que se puede establecer que este metal no representa un potencial peligro.

Guzmán-Colis et al. (2011), en el río San Pedro en Aguascalientes, documentaron que $25 \%$ de los sedimentos estuvieron moderadamente contaminados con este metal. En este estudio, el proceso de digestión fue el recomendado por la Agencia de Protección Ambiental de EE. UU., la US EPA. En otro estudio, el Cr se detectó en sedimentos de la costa este de EE. UU. en concentraciones de 3.8 a $130.9 \mathrm{\mu g} / \mathrm{g}$ en el año de 1994 y de 0.8 a $98.1 \mathrm{\mu g} / \mathrm{g}$ en 1995 (Hyland, Van Dolah, \& Snoots, 1999). Este metal se acumula en áreas donde se han establecido plantas de pinturas, como el mencionado por Schuck, Gevalt, Mullen, Menzie y Fogarty (2004), quienes encontraron altos niveles en sedimentos del Lago Waban, cercano a dicha planta.

Otro ejemplo es el nivel de $\mathrm{Cr}$ en sedimentos de un lago cercano a una zona industrial, donde se detectaron valores de hasta 3,712 $\mathrm{mg} \mathrm{kg}^{-1}$ (Environment Canada, 1998). En otro estudio, realizado en el río Turag en Bangladesh, los investigadores Banu, Chowdhury, Hossain y Nakagami (2013) encontraron concentraciones de $\mathrm{Cr}$ en un rango de 32.00-75.50 $\mathrm{mg} \mathrm{kg}^{-1}$. Para la lectura de metales se separaron $5 \mathrm{~g}$ de sedimentos y se realizó una digestión ácida con $\mathrm{HNO}_{3}$.

Las concentraciones de $\mathrm{Fe}$ en los sedimentos no rebasaron los criterios establecidos por la US EPA; es decir, están dentro del rango de no contaminado: hasta $17,000 \mathrm{mg} \mathrm{kg}^{-1}$. Es importante mencionar que el método de peróxido fue el que detectó una mayor cantidad con 4,408 $\mathrm{mg} \mathrm{kg}^{-1}$. Rubio Arias, Sau- cedo, Wood, Nuñez y Jiménez (2005b) encontraron niveles de 5.14 ppm como promedio en seis puntos aleatorios de un área ribereña en el río Conchos, cercano a la zona de presente estudio. Este estudio utilizó un método de digestión con la aplicación de agua regia. En otro estudio, Márquez, Senior, Martínez, Castañeda y González (2008) encontraron valores en los sedimentos de la laguna de Castillero, Venezuela, que oscilaron entre $1,821.58 \mu \mathrm{g} / \mathrm{g}$ y $3,768.42 \mu \mathrm{g} / \mathrm{g}$, con un promedio de $3,768.42 \mu \mathrm{g} / \mathrm{g}$. Para el análisis de metales, estos investigadores condujeron una digestión ácida con una mezcla en proporción 3:1:1 de $\mathrm{HNO}_{3}, \mathrm{HCl}$ y $\mathrm{HClO}_{4}$.

Para el elemento Li actualmente no existe normatividad que establezca límites máximos permisibles (LMP) en sedimentos o suelos. En el presente estudio, el método de peróxido detectó niveles con una media de $9.42 \mathrm{mg} \mathrm{kg}^{-1}$. Se calcula que en México desde la década de los noventa se han generado aproximadamente $77+$ por el uso y desecho de baterías. Debido a su baja adsorción, este metal puede lixiviarse fácilmente a los mantos freáticos, por lo que se ha encontrado en pequeñas cantidades en peces (Castro Díaz \& Díaz Arias, 2004). Se debe puntualizar que este elemento ha sido ampliamente utilizado en el tratamiento de desórdenes mentales como esquizofrenia y trastorno bipolar (Mohandas \& Rajmohan, 2007). No obstante, su uso para el tratamiento bipolar puede ocasionar problemas renales en adultos mayores (Anónimo, 2010). Es importante mencionar que el cuerpo acuático bajo estudio recibe las descargas provenientes de las ciudades de Cuauhtémoc y Anáhuac, las cuales pueden estar aportando este metal.

Para el elemento $\mathrm{Mg}$ no existe normatividad que establezca los LMP para las concentraciones en sedimentos o suelos. Los suelos contienen menos Mg que Ca debido a que el Mg no es tan absorbido como el Ca por los coloides del suelo y puede llegar a perderse más fácilmente por lixiviación (Chuchuca, 2014). Este elemento es esencial, incluso en grandes concentraciones en las células de las plantas, ya que es utilizado en reacciones enzimáticas y en la formación de ribosomas (Andrews \& Siccama, 1995). Las concentraciones de Mn no rebasaron los criterios establecidos por la US EPA, los cuales establecen un límite de $300 \mathrm{mg} \mathrm{kg}^{-1}$ para considerarlo como contaminado, en donde la media de peróxido más alta es de $138 \mathrm{mg} \mathrm{kg}^{-1}$.

Prego, Belzunce-Segarra, Helios-Rybicka y Barciela (1999) encontraron altas concentraciones en 
IIVESTIGACIÓn Y CIERCIA DE LA UNIVERSIDAD AUTÓNOMA DE AGUASCALIERTES sedimentos del río Ulla, España, con niveles de 2,1 10 mg kg-1 donde los puntos con mayores concentraciones se encontraron cercanos a fuentes industriales y descargas de aguas residuales con valores de $720 \mathrm{mg} \mathrm{kg}^{-1}$. Las concentraciones mínimas estuvieron en un rango de 122-174 $\mathrm{mg} \mathrm{kg}^{-1}$. Estos investigadores realizaron una digestión ácida con una mezcla de $\mathrm{HF}$ y agua regia $\left(\mathrm{HNO}_{3}-\mathrm{HCl}\right)$. Otro estudio en Saco del Golfo de Cariaco, Venezuela, encontró concentraciones por debajo de $200 \mathrm{mg} \mathrm{kg}^{-1}$ y la distribución de los metales fue relativamente uniforme. Las mayores concentraciones fueron en zonas con altos contenidos de limo, menor profundidad y donde los metales tienden a asociarse al grano fino (Lin et al., 2016; Sanguinetti, 2009).

Con respecto a las concentraciones de $\mathrm{Ni}$, no rebasaron los criterios establecidos por la USEPA, que establecen un límite de $20 \mathrm{mg} \mathrm{kg}^{-1}$ para considerarse contaminado y, además, no sobrepasaron las concentraciones de referencia totales establecidos en la NOM-147-SEMARNAT/SSA 1-2004, que son de $1.600 \mathrm{mg} \mathrm{kg}^{-1}$ para uso agrícola, residencial o comercial. En un estudio realizado por Fuentes Hernández (2001) en las lagunas de Chacopata (área rural) y de Los Patos (urbana) en Sucre, se encontró que la laguna de Los Patos presentó mayores concentraciones de $\mathrm{Ni}$ (13.66-38.05 $\mathrm{kg} \mathrm{g}^{-1}$ ) que la Chacopata, que obtuvo niveles de 7.81-44.43 $\mu \mathrm{g} \mathrm{g}^{-1}$.

Para la lectura de metales se utilizó una digestión con $\mathrm{HNO}_{3}$ y en forma posterior se agregó $\mathrm{H}_{2} \mathrm{O}_{2}$, hasta obtener $25 \mathrm{ml}$ de solución. En otro estudio, Martínez (2002) encontró concentraciones que oscilaron entre 2.01-49.87 $\mathrm{mg} \mathrm{kg}^{-1}$ en sedimentos del Golfo de Cariaco. Este autor atribuyó sus resultados a que los metales pesados pueden estar formando parte de la constitución de los minerales presentes en la zona y fueron transportados por las escorrentías en la época de lluvia y por los vientos que soplan en la región a lo largo de todo el año. Lin et al. (2016) especificaron que gran parte de los contaminantes presentes en sedimentos se deben a corrientes de aire que arrastran los metales pesados hacia diferentes cuerpos de agua.

Para el caso del Zn, Lin et al. (2016) mencionaron que la principal fuente en sedimentos proviene de la deposición atmosférica. En otro estudio, Zhang et al. (2016) encontraron valores en sedimentos del Lago Ponchartrain dentro del rango de 23.17-53.03 $\mathrm{mg} \mathrm{kg}^{-1}$. En las guías de Calidad del Medio Ambiente de Canadá, dentro de las directrices de calidad de sedimentos (ISQGS), se establece que los niveles probables de afectación biológicos para el Zn son de 123 $\mathrm{mg} \mathrm{kg}^{-1}$ en aguas dulces. Por tanto, basados en estas recomendaciones, los niveles en la laguna bajo estudio no representan algún riesgo biológico. Además, estos resultados no rebasaron los rangos establecidos por la US EPA, donde se considera a un ecosistema como moderadamente contaminado, cuando las concentraciones son superiores a $90 \mathrm{mg} \mathrm{kg}^{-1}$.

En un estudio realizado por Guzmán-Colis et al. (2011) en el río San Pedro en Aguascalientes, se encontró que la principal problemática de contaminación en los sedimentos fue por As, Cu y Zn, seguido de $\mathrm{Pb}$ y $\mathrm{Cr}$, pero es importante destacar que $50 \%$ de los sitios se encontraron contaminados por Zn (>200 $\mathrm{mg} \mathrm{kg}^{-1}$ ) e incluso dos sitios superaron los $800 \mathrm{mg} \mathrm{kg}^{-1}$, considerado por la PROFEPA como riesgo ecológico. Actualmente, no existe normatividad mexicana que establezca los LMP para las concentraciones de Zn en sedimentos o suelos. Banu et al. (2013) encontraron concentraciones en un rango de 94.60-190.10 $\mathrm{mg} \mathrm{kg}^{-1}$ en sedimentos en el río Turag en Bangladesh.

Con respecto al elemento Co, no existe normatividad mexicana e internacional que establezca los LMP en sedimentos o suelos. Palanques, Plana y Maldonado (1990) encontraron máximas concentraciones de 19.6 ppm de Co en la desembocadura del río Besos a una profundidad de $30 \mathrm{~cm}$, donde los niveles naturales se encuentran en valores de 5-6 p.p.m., por lo que se considera que hubo impacto antropogénico que elevó los niveles en sus sedimentos. En otro estudio, Sarria-Sacasa y Lacayo-Membreño (1997) encontraron que la concentración máxima de Co fue de $26.59 \mathrm{mg} \mathrm{kg}^{-1}$ en el lago Cocibolca, Nicaragua. La concentración máxima encontrada en los sedimentos del presente estudio fue de $2.64 \mathrm{mg}$ $\mathrm{kg}^{-1}$ para el peróxido y de $2.17 \mathrm{mg} \mathrm{kg}^{-1}$ para el agua regia, por lo que los niveles de este metal pueden ser considerados como bajos.

Las concentraciones de Cu no rebasaron los criterios de la US EPA, que establecen un límite de 25 $\mathrm{mg} \mathrm{kg}^{-1}$ para considerarse como contaminado. En las guías de Calidad del Medio Ambiente de Canadá, dentro de las directrices de calidad de sedimentos (ISQGs), se establece que los niveles probables de afectación biológica son de $197 \mathrm{mg} \mathrm{kg}^{-1}$ en aguas dulces y de $108 \mathrm{mg} \mathrm{kg}^{-1}$ para aguas marinas, los cuales en el presente estudio no llegaron a esas concentraciones. 


\section{IIVESTIGAGIÓn Y CIERCIA DE LA UחIVERSIDAD AUTÓNOMA DE AGUASCALIERTES}

En un estudio realizado por Martínez (2002) en el Golfo de Cariaco, Venezuela, se encontraron concentraciones que estuvieron en un rango de 1.72$48.30 \mathrm{\mu g} / \mathrm{g}$ en la Costa Norte, mientras que en el río Turag, Bangladesh, los investigadores Banu et al. (2013) notaron niveles en un rango de 46.30 a 60.00 $\mathrm{mg} \mathrm{kg}^{-1}$, por lo que estos valores son superiores a los del presente estudio. El nivel del elemento $\mathrm{Pb}$ no rebasó los criterios establecidos por la US EPA, un límite de $90 \mathrm{mg} \mathrm{kg}^{-1}$ para considerar un ecosistema como contaminado. Además, no sobrepasaron las concentraciones de referencia totales establecidas en la NOM-147-SEMARNAT/SSA1-2004 de $1.60 \mathrm{mg} \mathrm{kg}^{-1}$ para uso agrícola, residencial o comercial.

Márquez et al. (2008) encontraron concentraciones de $\mathrm{Pb}$ en la Laguna de Castilleros, Venezuela, de $17.02 \mathrm{mg} \mathrm{kg}^{-1}$, donde se apreciaron variaciones significativas en el sedimento. En otro estudio, Rubio Arias et al. (2006) encontraron niveles de $75.99 \mathrm{mg}$ $\mathrm{kg}^{-1}$ en sedimentos superficiales $\mathrm{y}$, además, reportaron concentraciones de $33.49 \mathrm{mg} \mathrm{kg-1}$ en áreas agrícolas aledañas a este ecosistema acuático. Esta diferencia puede explicarse por la época de muestreo, ya que este cuerpo acuático puede llegar a secarse y debido a los fuertes vientos se presenta una fuerte movilización de metales. Mapanda, Mangwayana, Nyamangara y Giller (2005) notaron niveles de $59.0 \mathrm{mg} \mathrm{kg}^{-1}$ de $\mathrm{Pb}$ en suelos superficiales $(0-20$ $\mathrm{cm}$ ) irrigados con aguas residuales. En sedimentos del río Turag en Bangladesh, los investigadores Banu et al. (2013) encontraron valores de $\mathrm{Pb}$ en un rango de $28.30-36.40 \mathrm{mg} \mathrm{kg}^{-1}$, mayores a los niveles encontrados en los sedimentos de la laguna bajo estudio.

Para el elemento Sr no existe normatividad que establezca los LMP en sedimentos o suelos. En un estudio realizado por Salazar, Lizano y Alfaro (2004) se determinaron las concentraciones de metales en sedimentos de las zonas costeras de Costa Rica, donde los niveles de $\mathrm{Sr}$ variaron de 7 a $20 \mathrm{mg} \mathrm{kg}^{-1}$. Los niveles máximos encontrados en los sedimentos del cuerpo acuático bajo estudio fueron de 176.77 $\mathrm{mg} \mathrm{kg}^{-1}$ con el método de peróxido y de $151.91 \mathrm{mg}$ $\mathrm{kg}^{-1}$ para el de agua regia.

CONCLUSIONES

Se concluye que los sedimentos de la Laguna de Bustillos no representan un riesgo potencial para el ecosistema (figura 5) en razón de que los niveles de metales no rebasan los límites establecidos, tanto en la US EPA, como en la Guía de Calidad del Medio Ambiente de Canadá y en la NOM-147-SEMARNAT/ SSA 1-2004. Las concentraciones no variaron significativamente en las dos profundidades y, en general, los niveles fueron bajos, por lo que se recomienda realizar estudios a mayor profundidad (figura 6) para evaluar si se ha presentado movilidad de metales y si ha habido interacción con la materia orgánica formando otros compuestos. Se recomienda realizar un estudio específico en condiciones de laboratorio mediante concentraciones conocidas en sedimentos, con el propósito de validar cuál método de digestión pondera más acertadamente la realidad.

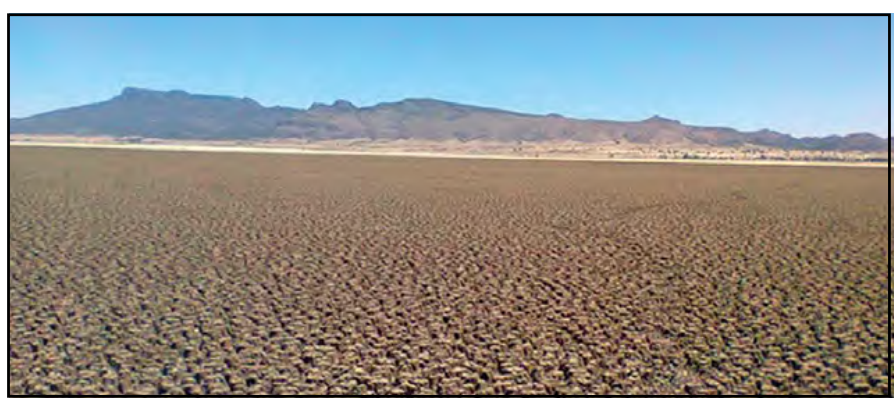

Figura 5. Los sedimentos encontrados en la Laguna de Bustillos no representan un riesgo potencial para el ecosistema.

Fotografía del equipo de investigación.

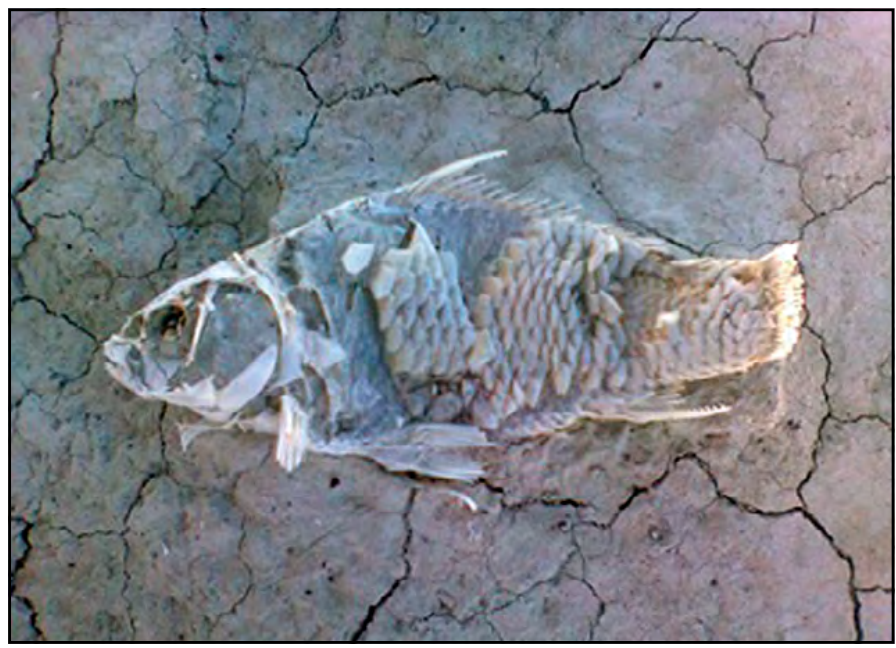

Figura 6. Se recomienda realizar estudios a mayor profundidad para evaluar si se ha presentado movilidad de metales y si ha habido interacción con la materia orgánica. Fotografía del equipo de investigación. 
- Alemayehu, T. (2006). Heavy metal concentration in the urban environment of Addis Ababa, Ethiopia. Soil \& Sediment Contamination: An International Journal, 15(6), 591-602. doi: $10.1080 / 15320380600959081$

- Amado A., J. P., Ortiz, P., Solano, V., Orozco, F., \& Ramírez, V. (1991). Calidad del agua y el suelo para uso agropecuario en la región aledaña a la Laguna de Bustillos, Chih. En J. L. Tovar \& R. Quintero (Eds.), La Investigación Edafológica en México 1990-1991. Memorias del XXIV Congreso Nacional de la Sociedad Mexicana de la Ciencia del Suelo (p. 225). Pachuca, Hidalgo, México.

- Andrews, J. A., \& Siccama, T. G. (1995). Retranslocation of calcium and magnesium at the heartwood-sapwood boundary of Atlantic white cedar. Ecology, 76(2), 659-663. doi: $10.2307 / 1941225$

- Anónimo. (2010). Lithium. Renal failure and nephrotic syndrome: Case report. Reactions Weekly, 1291(1), 32. doi: 10.2165/00128415-201012910-00097

- Banu, Z., Chowdhury, S. A., Hossain, D., \& Nakagami, K. (2013). Contamination and ecological risk assessment of heavy metal in the sediment of Turag River, Bangaldesh: An Index Analysis Aprproach. Journal for Water Resource and Protection (JWARP), 5(2), 28446. doi: 10.4236/jwarp.2013.52024

- Canadian Council of Ministers of the Environment. (2002). Canadian water quality guidelines for the protection of aquatic life: Total particulate matter. En Canadian Environmental Quality Guidelines, 1999 (Actualizado en 2002, 18 pp.). Winnipeg, MB: Autor.

- Castro Díaz, J., \& Díaz Arias, M. L. (2004). La contaminación por pilas y baterías en México. México: Instituto Nacional de Ecología y Cambio Climático. Recuperado el 13 de octubre de 2014, de http//:www2.inecc.gob.mx/publicaciones/libros/438/cap5. html

- Chen, M., Ding, S., Zhang, L., Li, Y., Sun, Q., \& Zhang, C. (2017). An investigation of the effects of elevated phosphorus in water on the release of heavy metals in sediments at a high resolution. The Science of the Total Environment, 575, 330-337.

- Chuchuca, M. N. N. (2014). Mapificación del grado de fertilidad de suelos, mediante los sistemas de información geográfica (SIG), del cantón las lajas provincia de el oro (Tesis de licenciatura). Ecuador: Universidad Técnica De Machala.

- Comisión Nacional del Agua. (s. f.). Actualización de la disponibilidad media anval de agua en el acuífero Cuauhtémoc (0805), estado de Chihuahua [Compilación de documentos alusivos en pdf]. Recuperada el 22 de abril de 2017, de https:// www.gob.mx/cms/uploads/attachment/file/103566/DR_0805. pdf
- Environment Canada. (1998). Canadian sediment quality guidelines for chromium: Supporting document [Draft]. Ottawa: Environmental Conservation Service, Ecosystem Science Directorate. Science Policy and Environmental Quality Branch, Guidelines and Standards Division.

- Fuentes Hernández, M. V. (2001). Estudio geoquímico de carbohidratos, hidrocarburos, aceites y grasas en la Laguna de Chacopata, estado Sucre, Venezuela. Boletín del Instituto Oceanográfico de Venezuela, 40(2), 31-37.

- García, F. M., \& Maza, J. A. (1998). Origen y propiedades de los sedimentos. En Manual de Ingeniería de Ríos (Cap. 7). México: UNAM.

- Guzmán-Colis, G., Ramírez-López, E. M., Thalasso, F., RodríguezNarciso, S., Guerrero-Barrera, A. L., \& Avelar-González, F. J. (2011). Evaluación de contaminantes en agua y sedimentos del río San Pedro en el estado de Aguascalientes. Universidad y Ciencia, 27(1), 17-32. Recuperado el 20 de octubre de 2014, de http:// www.universidadyciencia.ujat.mx/index.php? $\mid \mathrm{D}=24 \&$ art=463

- Hyland, J. L., Van Dolah, R. F., \& Snoots, T. R. (1999). Predicting stress in benthic communities of southeastern U.S. estuaries in relation to chemical contamination of sediments. Environmental Toxicology and Chemistry, 18(11), 2557-2564.

- Lin, Q., Liu, E., Zhang, E., Li, K., \& Shen, J. (2016). Spatial distribution, contamination and ecological risk assessment of heavy metals in surface sediments of Erhai Lake, a large eutrophic plateau lake in southwest China. Catena, 145, 193-203.

- Mapanda, F., Mangwayana, E. N., Nyamangara, J., \& Giller, K. E. (2005). The effect of long-term irrigation using wastewater on heavy metal contents of soils under vegetables in Harare, Zimbabwe. Agriculture, Ecosystems \& Environment, 107(2-3), 151165.

- Márquez, A., Senior, W., Martínez, G., Castañeda, J., \& González, A. (2008). Concentraciones de metales en sedimentos y tejidos musculares de algunos peces de la laguna de Castillero, Venezuela. Revista Científica, FCV-LUZ, 18(2), 121-133.

- Martínez, G. (2002). Metales pesados en sedimentos superficiales del Golfo de Cariaco, Venezuela. Boletín del Instituto Oceanográfico de Venezuela, 41 (1-2), 83-86.

- Mohandas, E., \& Rajmohan, V. (2007). Lithium use in special populations. Indian Journal of Psychiatry, 49(3), 21 1-218.

- Ochoa-Rivero, J. M., Reyes-Fierro, A. V., Peralta-Pérez, M. R., Zavala-Díaz, F. J., Ballinas-Casarubias, L., Salmerón, I.,...RochaGutiérrez, B. A. (2017). Levels and distribution of pollutants in the waters of an aquatic ecosystem in Northern Mexico. International Journal of Environmental Research and Public Health, 14(5), 456. doi: 10.3390/ijerph14050456 
- Páez, F. (1996). Fuentes de metales en la zona costera marina. En A. V. Botello, J. L. Rojas, J. A. Benítez, \& D. Zárate (Eds.), Golfo de México. Contaminación e impacto ambiental: Diagnóstico, y tendencias (EPOMEX Serie científica 297-308). México: Universidad Autónoma de Campeche.

- Palanques, A., Plana, F., \& Maldonado, A. (1990). Recent influence of man on the Ebro margin sedimentation system, northwestern Mediterranean Sea. Marine Geology, 95(3-4), 247263.

- Prego, R., Belzunce-Segarra, M. J., Helios-Rybicka, E., \& Barciela, M. C. (1999). Cadmium, manganese, nickel and lead contents in surface sediments of the lower Ulla River and its estuary (northwest Spain). Boletín-Instituto Español de Oceanografía, 15(1-4), 495-500.

- Rodríguez, S. V. (2013). Niveles de contaminación por metales pesados en la especie Cyprinus carpio (carpa común) de la Laguna de Bustillos, México (Tesis de licenciatura). Universidad Autónoma de Chihuahua, México.

- Rubio Arias, H. O., \& Jiménez, J. A. (2012). Estadística aplicada con análisis en Minitab (169pp.). México: Universidad Autónoma de Chihuahua.

- Rubio Arias, H. O., Saucedo, R., Bautista, M. R., Wood, K., Holguin, C., \& Jiménez, J. (2006). Are crop and range land being contaminated with cadmium and lead in sediments transported by wind from an adjacent contaminated shallow lake? En WIT Transactions on Ecology and the Environment: Vol. 89. Geo-Environment and Landscape Evolution II (pp. 135-141). doi: 10.2495/GEO060141

- Rubio Arias, H. O., Saucedo, R., Lara, C. R., Wood, K., \& Jiménez, J. (2005a). Water quality in the Laguna de Bustillos, Chihuahua, Mexico. En WIT Transactions on Ecology and the Environment: Vol. 80. Water Resources Management III (pp. 155-160). doi: 10.2495/WRM050161

- Rubio Arias, H. O., Saucedo, R., Wood, K., Nuñez, A., \& Jiménez, J. (2005b). Metal contamination of a riparian area in the Conchos watershed of Chihuahua, Mexico. En WIT Transactions on Ecology and the Environment: Vol. 80. Water Resources Management III (pp. 269-275). doi: 10.2495/WRM050281
- Salazar, M. A., Lizano, O. G., \& Alfaro, E. J. (2004). Composición de sedimentos en las zonas costeras de Costa Rica utilizando fluorescencia de rayos X (FRX). Revista de Biología Tropical, 52 (supl. 2), 61-75.

- Sanguinetti, G. O. A. (2009). Evaluación de los métodos propuestos por Campanella y BCR utilizados para la extracción secuencial de metales pesados en sedimentos y posibles efectos tóxicos de estos sedimentos (Tesis). Universidad de Oriente-Núcleo de Sucre, Venezuela.

- Sarria-Sacasa, K., \& Lacayo-Membreño, J. (1997). Determinación de metales pesados en sedimento del lago Cocibolca, Nicaragua [pdf]. Recuperado el 2 de noviembre de 2014, de http://www.bvsde.org.ni/Web_textos/CIRA/Cira0005/15.pdf

- Schuck, R., Gevalt, D., Mullen, J., Menzie, C., \& Fogarty, K. (2004). Risk-based remediation of lead and chromium impacted sediments in Lake Waban, Wellesley, MA: A case study. Soil \& Sediment Contamination. Recuperado el 2 de noviembre de 2014, de http://www.highbeam.com/doc/1P3-618978941. html

- $\quad$ Secretaría de Medio Ambiente y Recursos Naturales. (2007). NOM-147-SEMARNAT/SSA 1-2004. Que establece criterios para determinar las concentraciones de remediación de suelos contaminados por arsénico, bario, berilio, cadmio, cromo hexavalente, mercurio, níquel, plata, plomo, selenio, talio y/o vanadio. Diario Oficial de la Federación, 2 de marzo de 2007.

- Sprovieri, M., Feo, M., Prevedello, L., Manta, D. S., Sammartino, S., Tamburrino, S., \& Marsella, E. (2007). Heavy metals, polycyclic aromatic of the Naples harbour (southern Italy). Chemosphere, 67(5), 998-1009.

- White, W. J., Morris, L. A., Warnell, D. B., Pinho, A. P., Jackson, C. R., \& West, L. T. (2007). Sediment retention by forested filter strips in the Piedmont of Georgia (Report). Journal of Soil and Water Conservation, 62(6), 453-463. Recuperado el 5 de noviembre de 2014, de http://www.highbeam.com/doc/1G1-172427311. html

- Zhang, Z., Wang, J. J., Ali, A., \& DeLaune, R. D. (2016). Heavy metals and metalloid contamination in Lovisiana Lake Pontchartrain Estuary along 1-10 Bridge. Transportation Research Part D: Transport and Environment, 44, 66-77. 